\title{
Discrete matrix delayed exponential for two delays and its property
}

\author{
Josef Diblík ${ }^{1,2^{*}}$ and Blanka Morávková2,3
}

"Correspondence:

diblik.j@fce.vutbr.cz;

diblik@feec.vutbr.cz

'Department of Mathematics and

Descriptive Geometry, AdMaS,

Faculty of Civil Engineering, Brno

University of Technology, Brno,

Czech Republic

${ }^{2}$ Department of Mathematics,

Faculty of Electrical Engineering and

Communication, Brno University of

Technology, Brno, Czech Republic

Full list of author information is

available at the end of the article

\begin{abstract}
In recent papers, a discrete matrix delayed exponential for a single delay was defined and its main property connected with the solution of linear discrete systems with a single delay was proved. In the present paper, a generalization of the concept of discrete matrix delayed exponential is designed for two delays and its main property is proved as well.
\end{abstract}

\section{Introduction}

Throughout the paper, we use the following notation. For integers $s, t, s \leq t$, we define a set $\mathbb{Z}_{s}^{t}:=\{s, s+1, \ldots, t-1, t\}$. Similarly, we define sets $\mathbb{Z}_{-\infty}^{t}:=\{\ldots, t-1, t\}$ and $\mathbb{Z}_{s}^{\infty}:=\{s, s+$ $1, \ldots\}$. The function $\lfloor\cdot\rfloor$ used below is the floor integer function. We employ the following property of the floor integer function:

$$
x-1<\lfloor x\rfloor \leq x,
$$

where $x \in \mathbb{R}$.

Define binomial coefficients as customary, i.e., for $n \in \mathbb{Z}$ and $k \in \mathbb{Z}$,

$$
\left(\begin{array}{l}
n \\
k
\end{array}\right):= \begin{cases}\frac{n !}{k !(n-k) !} & \text { if } n \geq k \geq 0 \\
0 & \text { otherwise }\end{cases}
$$

We recall that for a well-defined discrete function $f(k)$, the forward difference operator $\Delta$ is defined as $\Delta f(k)=f(k+1)-f(k)$. In the paper, we also adopt the customary notation $\sum_{i=i_{1}}^{i_{2}} g_{i}=0$ if $i_{2}<i_{1}$. In the case of double sums, we set

$$
\sum_{i=i_{1}, j=j_{1}}^{i_{2}, j_{2}} g_{i j}=0
$$

if at least one of the inequalities $i_{2}<i_{1}, j_{2}<j_{1}$ holds.

In $[1,2]$, a discrete matrix delayed exponential for a single delay $m \in \mathbb{N}$ was defined as follows.

\section{Springer}

○ 2013 Diblík and Morávková; licensee Springer. This is an Open Access article distributed under the terms of the Creative Commons Attribution License (http://creativecommons.org/licenses/by/2.0), which permits unrestricted use, distribution, and reproduction in any medium, provided the original work is properly cited. 
Definition 1 For an $r \times r$ constant matrix $B, k \in \mathbb{Z}$, and fixed $m \in \mathbb{N}$, we define the discrete matrix delayed exponential $\mathrm{e}_{m}^{B k}$ as follows:

$$
\mathrm{e}_{m}^{B k}:= \begin{cases}\Theta & \text { if } k \in \mathbb{Z}_{-\infty}^{-m-1}, \\
I+\sum_{j=1}^{\ell} B^{j} \cdot\left(\begin{array}{c}
k-m(j-1) \\
j
\end{array}\right) & \text { if } \ell=0,1,2, \ldots, k \in \mathbb{Z}_{(\ell-1)(m+1)+1}^{\ell(m+1)}\end{cases}
$$

where $\Theta$ is an $r \times r$ null matrix and $I$ is an $r \times r$ unit matrix.

Next, the main property (Theorem 1 below) of discrete matrix delayed exponential for a single delay $m \in \mathbb{N}$ is proved in [1].

Theorem 1 Let $B$ be a constant $r \times r$ matrix. Then, for $k \in \mathbb{Z}_{-m}^{\infty}$,

$$
\Delta \mathbf{e}_{m}^{B k}=B \mathbf{e}_{m}^{B(k-m)} .
$$

The paper is concerned with a generalization of the notion of discrete matrix delayed exponential for two delays and a proof of one of its properties, similar to the main property (4) of discrete matrix delayed exponential for a single delay.

\section{Discrete matrix delayed exponential for two delays and its main property}

We define a discrete $r \times r$ matrix function $\mathrm{e}_{m n}^{B C k}$ called the discrete matrix delayed exponential for two delays $m, n \in \mathbb{N}, m \neq n$ and for two $r \times r$ commuting constant matrices $B$, $C$ as follows.

Definition 2 Let $B, C$ be constant $r \times r$ matrices with the property $B C=C B$ and let $m, n \in \mathbb{N}, m \neq n$ be fixed integers. We define a discrete $r \times r$ matrix function $\mathrm{e}_{m n}^{B C k}$ called the discrete matrix delayed exponential for two delays $m, n$ and for two $r \times r$ constant matrices $B, C$ :

$$
\mathrm{e}_{m n}^{B C k}:= \begin{cases}\Theta & \text { if } k \in \mathbb{Z}_{-\infty}^{-\max \{m, n\}-1}, \\
I & \text { if } k \in \mathbb{Z}_{-\max \{m, n\}}^{0}, \\
I+(B+C) \sum_{i=0, j=0}^{p_{(k)}-1, q_{(k)}-1} B^{i} C^{j}\left(\begin{array}{c}
i+j \\
i
\end{array}\right)\left(\begin{array}{c}
k-m i-n j \\
i+j+1
\end{array}\right) & \text { if } k \in \mathbb{Z}_{1}^{\infty},\end{cases}
$$

where

$$
p_{(k)}:=\left\lfloor\frac{k+m}{m+1}\right\rfloor, \quad q_{(k)}:\left\lfloor\left\lfloor\frac{k+n}{n+1}\right\rfloor .\right.
$$

The main property of $\mathrm{e}_{m n}^{B C k}$ is given by the following theorem.

Theorem 2 Let $B, C$ be constant $r \times r$ matrices with the property $B C=C B$ and let $m, n \in \mathbb{N}$, $m \neq n$ be fixed integers. Then

$$
\Delta \mathbf{e}_{m n}^{B C k}=B \mathrm{e}_{m n}^{B C(k-m)}+C \mathrm{e}_{m n}^{B C(k-n)}
$$

holds for $k \geq 0$. 
Proof Let $k \geq 1$. From (1) and (5), we can see easily that, for an integer $k \geq 0$ satisfying

$$
\left(p_{(k)}-1\right)(m+1)+1 \leq k \leq p_{(k)}(m+1) \wedge\left(q_{(k)}-1\right)(n+1)+1 \leq k \leq q_{(k)}(n+1),
$$

the relation

$$
\Delta \mathbf{e}_{m n}^{B C k}=\Delta\left[I+(B+C) \sum_{i=0, j=0}^{p_{(k)}-1, q_{(k)}-1} B^{i} C^{j}\left(\begin{array}{c}
i+j \\
i
\end{array}\right)\left(\begin{array}{c}
k-m i-n j \\
i+j+1
\end{array}\right)\right]
$$

holds in accordance with Definition 2 of $\mathrm{e}_{m n}^{B C k}$. Since $\Delta I=\Theta$, we have

$$
\Delta \mathrm{e}_{m n}^{B C k}=\Delta\left[(B+C) \sum_{i=0, j=0}^{p_{(k)}-1, q_{(k)}-1} B^{i} C^{j}\left(\begin{array}{c}
i+j \\
i
\end{array}\right)\left(\begin{array}{c}
k-m i-n j \\
i+j+1
\end{array}\right)\right] .
$$

Considering the increment by its definition, i.e.,

$$
\Delta \mathrm{e}_{m n}^{B C k}=\mathrm{e}_{m n}^{B C(k+1)}-\mathrm{e}_{m n}^{B C k},
$$

we conclude that it is reasonable to divide the proof into four parts with respect to the value of integer $k$. In case one, $k$ is such that

$$
\left(p_{(k)}-1\right)(m+1)+1 \leq k<p_{(k)}(m+1) \wedge\left(q_{(k)}-1\right)(n+1)+1 \leq k<q_{(k)}(n+1),
$$

in case two

$$
k=p_{(k)}(m+1) \wedge\left(q_{(k)}-1\right)(n+1)+1 \leq k<q_{(k)}(n+1),
$$

in case three

$$
\left(p_{(k)}-1\right)(m+1)+1 \leq k<p_{(k)}(m+1) \wedge k=q_{(k)}(n+1)
$$

and in case four

$$
k=p_{(k)}(m+1) \wedge k=q_{(k)}(n+1) .
$$

We see that the above cases cover all the possible relations between $k, p_{(k)}$ and $q_{(k)}$.

In the proof, we use the identities

$$
\left(\begin{array}{c}
n+1 \\
k
\end{array}\right)=\left(\begin{array}{l}
n \\
k
\end{array}\right)+\left(\begin{array}{c}
n \\
k-1
\end{array}\right)
$$

where $n, k \in \mathbb{N}$ and

$$
\left(\begin{array}{l}
i \\
i
\end{array}\right)=\left(\begin{array}{c}
i-1 \\
i-1
\end{array}\right), \quad\left(\begin{array}{c}
j \\
0
\end{array}\right)=\left(\begin{array}{c}
j-1 \\
0
\end{array}\right), \quad\left(\begin{array}{c}
i+j \\
i
\end{array}\right)=\left(\begin{array}{c}
i+j-1 \\
i-1
\end{array}\right)+\left(\begin{array}{c}
i+j-1 \\
i
\end{array}\right),
$$

where $i, j \in \mathbb{N}$, which are derived from (2) and (9). 
I. $\left(p_{(k)}-1\right)(m+1)+1 \leq k<p_{(k)}(m+1) \wedge\left(q_{(k)}-1\right)(n+1)+1 \leq k<q_{(k)}(n+1)$

From (1) and (5), we get

$$
\begin{aligned}
& p_{(k-m)}=\left\lfloor\frac{k-m+m}{m+1}\right\rfloor \leq \frac{k}{m+1}<p_{(k)}, \\
& p_{(k-m)}=\left\lfloor\frac{k-m+m}{m+1}\right\rfloor>\frac{k}{m+1}-1=\frac{k-m-1}{m+1}>p_{(k)}-2 .
\end{aligned}
$$

Therefore, $p_{(k-m)}=p_{(k)}-1$ and, by Definition 2,

$$
\mathrm{e}_{m n}^{B C(k-m)}=I+(B+C) \sum_{i=0, j=0}^{p_{(k)}-2, q_{(k-m)}-1} B^{i} C^{j}\left(\begin{array}{c}
i+j \\
i
\end{array}\right)\left(\begin{array}{c}
k-m-m i-n j \\
i+j+1
\end{array}\right) .
$$

Similarly, omitting details, we get (using (1), and (5)) $q_{(k-n)}=q_{(k)}-1$ and

$$
\mathrm{e}_{m n}^{B C(k-n)}=I+(B+C) \sum_{i=0, j=0}^{p_{(k-n)}-1, q_{(k)}-2} B^{i} C^{j}\left(\begin{array}{c}
i+j \\
i
\end{array}\right)\left(\begin{array}{c}
k-n-m i-n j \\
i+j+1
\end{array}\right) .
$$

Let $q_{(k-m)} \geq 1$. We show that

$$
\left(\begin{array}{c}
k-m-m i-n j \\
i+j+1
\end{array}\right)=0 \quad \text { if } i \geq 0, j \geq q_{(k-m)}
$$

In accordance with (1),

$$
q_{(k-m)}=\left\lfloor\frac{k-m+n}{n+1}\right\rfloor>\frac{k-m+n}{n+1}-1=\frac{k-m-1}{n+1}
$$

or

$$
k-m<(n+1) q_{(k-m)}+1 \leq(m+1) i+(n+1) j+1 \quad \text { if } i \geq 0, j \geq q_{(k-m)} .
$$

From the last inequality, we get

$$
k-m-m i-n j<i+j+1 \quad \text { if } i \geq 0, j \geq q_{(k-m)}
$$

and (13) holds by (2). For that reason and since $q_{(k-m)} \leq q_{(k)}$, we can replace $q_{(k-m)}$ by $q_{(k)}$ in (11). Thus, we have

$$
\mathrm{e}_{m n}^{B C(k-m)}=I+(B+C) \sum_{i=0, j=0}^{p_{(k)}-2, q_{(k)}-1} B^{i} C^{j}\left(\begin{array}{c}
i+j \\
i
\end{array}\right)\left(\begin{array}{c}
k-m(i+1)-n j \\
i+j+1
\end{array}\right) .
$$

It is easy to see that, due to (3), formula (14) can be used instead of (11) if $q_{(k-m)}<1$ also.

Let $p_{(k-n)} \geq 1$. Similarly, we can show that

$$
\left(\begin{array}{c}
k-n-m i-n j \\
i+j+1
\end{array}\right)=0 \quad \text { if } i \geq p_{(k-n)}, j \geq 0
$$


and, since $p_{(k-n)} \leq p_{(k)}$, we can replace $p_{(k-n)}$ by $p_{(k)}$ in (12). Thus, we have

$$
\mathrm{e}_{m n}^{B C(k-n)}=I+(B+C) \sum_{i=0, j=0}^{p_{(k)}-1, q_{(k)}-2} B^{i} C^{j}\left(\begin{array}{c}
i+j \\
i
\end{array}\right)\left(\begin{array}{c}
k-m i-n(j+1) \\
i+j+1
\end{array}\right) .
$$

It is easy to see that, due to (3), formula (15) can be used instead of (12) if $p_{(k-n)}<1$, too.

Due to (1), we also conclude that

$$
p_{(k+1)}=p_{(k)}, \quad q_{(k+1)}=q_{(k)}
$$

because

$$
p_{(k+1)}=\left\lfloor\frac{k+1+m}{m+1}\right\rfloor \leq \frac{k}{m+1}+1<p_{(k)}+1
$$

and

$$
p_{(k+1)}=\left\lfloor\frac{k+1+m}{m+1}\right\rfloor>\frac{k+1+m}{m+1}-1=\frac{k}{m+1} \geq p_{(k)}-1+\frac{1}{m+1} .
$$

The second formula can be proved similarly.

Now we are able to prove that

$$
\begin{aligned}
\Delta \mathrm{e}_{m n}^{B C k}= & B \mathrm{e}_{m n}^{B C(k-m)}+C \mathrm{e}_{m n}^{B C(k-n)} \\
= & B\left[I+(B+C) \sum_{i=0, j=0}^{p_{(k)}-2, q_{(k)}-1} B^{i} C^{j}\left(\begin{array}{c}
i+j \\
i
\end{array}\right)\left(\begin{array}{c}
k-m(i+1)-n j \\
i+j+1
\end{array}\right)\right] \\
& +C\left[I+(B+C) \sum_{i=0, j=0}^{p_{(k)}-1, q_{(k)}-2} B^{i} C^{j}\left(\begin{array}{c}
i+j \\
i
\end{array}\right)\left(\begin{array}{c}
k-m i-n(j+1) \\
i+j+1
\end{array}\right)\right] .
\end{aligned}
$$

With the aid of (7), (8), (9) and (16), we get

$$
\begin{aligned}
\Delta \mathrm{e}_{m n}^{B C k}= & \mathrm{e}_{m n}^{B C(k+1)}-\mathrm{e}_{m n}^{B C k} \\
= & I+(B+C) \sum_{i=0, j=0}^{p_{(k+1)}-1, q_{(k+1)}-1} B^{i} C^{j}\left(\begin{array}{c}
i+j \\
i
\end{array}\right)\left(\begin{array}{c}
k+1-m i-n j \\
i+j+1
\end{array}\right) \\
& -I-(B+C) \sum_{i=0, j=0}^{p_{(k)}-1, q_{(k)}-1} B^{i} C^{j}\left(\begin{array}{c}
i+j \\
i
\end{array}\right)\left(\begin{array}{c}
k-m i-n j \\
i+j+1
\end{array}\right) \\
= & I+(B+C) \sum_{i=0, j=0}^{p_{(k)}-1, q_{(k)}-1} B^{i} C^{j}\left(\begin{array}{c}
i+j \\
i
\end{array}\right)\left(\begin{array}{c}
k+1-m i-n j \\
i+j+1
\end{array}\right) \\
& -I-(B+C) \sum_{i=0, j=0}^{p_{(k)}-1, q_{(k)}-1} B^{i} C^{j}\left(\begin{array}{c}
i+j \\
i
\end{array}\right)\left(\begin{array}{c}
k-m i-n j \\
i+j+1
\end{array}\right) \\
= & (B+C) \sum_{i=0, j=0}^{p_{(k)}-1, q_{(k)}-1} B^{i} C^{j}\left(\begin{array}{c}
i+j \\
i
\end{array}\right)\left[\left(\begin{array}{c}
k+1-m i-n j \\
i+j+1
\end{array}\right)-\left(\begin{array}{c}
k-m i-n j \\
i+j+1
\end{array}\right)\right]
\end{aligned}
$$




$$
\begin{aligned}
& =(B+C) \sum_{i=0, j=0}^{p_{(k)}-1, q_{(k)}-1} B^{i} C^{j}\left(\begin{array}{c}
i+j \\
i
\end{array}\right)\left(\begin{array}{c}
k-m i-n j \\
i+j
\end{array}\right) \\
& =(B+C)\left[I+\sum_{i=1}^{p_{(k)}-1} B^{i} C^{0}\left(\begin{array}{c}
i \\
i
\end{array}\right)\left(\begin{array}{c}
k-m i \\
i
\end{array}\right)+\sum_{j=1}^{q_{(k)}-1} B^{0} C^{j}\left(\begin{array}{l}
j \\
0
\end{array}\right)\left(\begin{array}{c}
k-n j \\
j
\end{array}\right)\right. \\
& \left.+\sum_{i=1, j=1}^{p_{(k)}-1, q_{(k)}-1} B^{i} C^{j}\left(\begin{array}{c}
i+j \\
i
\end{array}\right)\left(\begin{array}{c}
k-m i-n j \\
i+j
\end{array}\right)\right] .
\end{aligned}
$$

By (10), we have

$$
\begin{aligned}
\Delta \mathrm{e}_{m n}^{B C k}= & (B+C)\left[I+\sum_{i=1}^{p_{(k)}-1} B^{i} C^{0}\left(\begin{array}{c}
i-1 \\
i-1
\end{array}\right)\left(\begin{array}{c}
k-m i \\
i
\end{array}\right)\right. \\
& +\sum_{j=1}^{q_{(k)}-1} B^{0} C^{j}\left(\begin{array}{c}
j-1 \\
0
\end{array}\right)\left(\begin{array}{c}
k-n j \\
j
\end{array}\right) \\
& +\sum_{i=1, j=1}^{p_{(k)}-1, q_{(k)}-1} B^{i} C^{j}\left(\begin{array}{c}
i+j-1 \\
i-1
\end{array}\right)\left(\begin{array}{c}
k-m i-n j \\
i+j
\end{array}\right) \\
& \left.+\sum_{i=1, j=1}^{p_{(k)}-1, q_{(k)}-1} B^{i} C^{j}\left(\begin{array}{c}
i+j-1 \\
i
\end{array}\right)\left(\begin{array}{c}
k-m i-n j \\
i+j
\end{array}\right)\right] \\
= & (B+C)\left[\begin{array}{c}
I+p_{(k)}^{-1, q_{(k)}-1} B^{i} C^{j}\left(\begin{array}{c}
i+j-1 \\
i-1
\end{array}\right)\left(\begin{array}{c}
k-m i-n j \\
i+j
\end{array}\right) \\
\end{array}\right. \\
& \left.+\sum_{i=1, j=0} \sum_{(k, j)-1, q_{(k)}-1} B^{i} C^{j}\left(\begin{array}{c}
i+j-1 \\
i
\end{array}\right)\left(\begin{array}{c}
k-m i-n j \\
i+j
\end{array}\right)\right]
\end{aligned}
$$

Now in the first sum we replace the summation index $i$ by $i+1$ and in the second sum we replace the summation index $j$ by $j+1$. Then

$$
\begin{aligned}
\Delta \mathrm{e}_{m n}^{B C k}= & (B+C)\left[I+\sum_{i=0, j=0}^{p_{(k)}-2, q_{(k)}-1} B^{i+1} C^{j}\left(\begin{array}{c}
i+j \\
i
\end{array}\right)\left(\begin{array}{c}
k-m(i+1)-n j \\
i+j+1
\end{array}\right)\right. \\
& \left.+\sum_{i=0, j=0}^{p_{(k)}-1, q_{(k)}-2} B^{i} C^{j+1}\left(\begin{array}{c}
i+j \\
i
\end{array}\right)\left(\begin{array}{c}
k-m i-n(j+1) \\
i+j+1
\end{array}\right)\right] \\
= & B+B(B+C) \sum_{i=0, j=0}^{p_{(k)}-2, q_{(k)}-1} B^{i} C^{j}\left(\begin{array}{c}
i+j \\
i
\end{array}\right)\left(\begin{array}{c}
k-m(i+1)-n j \\
i+j+1
\end{array}\right) \\
& +C+C(B+C) \sum_{i=0, j=0}^{p_{(k)}-1, q_{(k)}-2} B^{i} C^{j}\left(\begin{array}{c}
i+j \\
i
\end{array}\right)\left(\begin{array}{c}
k-m i-n(j+1) \\
i+j+1
\end{array}\right) \\
= & B\left[I+(B+C) \sum_{i=0, j=0}^{p_{(k)}-2, q_{(k)}-1} B^{i} C^{j}\left(\begin{array}{c}
i+j \\
i
\end{array}\right)\left(\begin{array}{c}
k-m(i+1)-n j \\
i+j+1
\end{array}\right)\right]
\end{aligned}
$$




$$
\begin{aligned}
& +C\left[I+C(B+C) \sum_{i=0, j=0}^{p_{(k)}-1, q_{(k)}-2} B^{i} C^{j}\left(\begin{array}{c}
i+j \\
i
\end{array}\right)\left(\begin{array}{c}
k-m i-n(j+1) \\
i+j+1
\end{array}\right)\right] \\
= & B \mathrm{e}_{m n}^{B C(k-m)}+C \mathrm{e}_{m n}^{B C(k-n) .}
\end{aligned}
$$

Due to (14) and (15), we conclude that formula (17) is valid.

II. $k=p_{(k)}(m+1) \wedge\left(q_{(k)}-1\right)(n+1)+1 \leq k<q_{(k)}(n+1)$

In this case,

$$
\begin{aligned}
& p_{(k-m)}=\left\lfloor\frac{k-m+m}{m+1}\right\rfloor=\left\lfloor\frac{k}{m+1}\right\rfloor=p_{(k)}, \\
& p_{(k+1)}=\left\lfloor\frac{k+1+m}{m+1}\right\rfloor \leq \frac{k+1+m}{m+1}=\frac{k}{m+1}+1=p_{(k)}+1, \\
& p_{(k+1)}=\left\lfloor\frac{k+1+m}{m+1}\right\rfloor>\frac{k+1+m}{m+1}-1=\frac{k}{m+1}=p_{(k)}
\end{aligned}
$$

and $p_{(k+1)}=p_{(k)}+1$. In addition to this (see relevant computations performed in case $\mathrm{I}$ ), we have $q_{(k-n)}=q_{(k)}-1$ and $q_{(k+1)}=q_{(k)}$.

Then

$$
\begin{aligned}
& \mathrm{e}_{m n}^{B C k}=I+(B+C) \sum_{i=0, j=0}^{p_{(k)}-1, q_{(k)}-1} B^{i} C^{j}\left(\begin{array}{c}
i+j \\
i
\end{array}\right)\left(\begin{array}{c}
k-m i-n j \\
i+j+1
\end{array}\right), \\
& \mathrm{e}_{m n}^{B C(k+1)}=I+(B+C) \sum_{i=0, j=0}^{p_{(k)}, q_{(k)}-1} B^{i} C^{j}\left(\begin{array}{c}
i+j \\
i
\end{array}\right)\left(\begin{array}{c}
k+1-m i-n j \\
i+j+1
\end{array}\right)
\end{aligned}
$$

and

$$
\begin{aligned}
& \mathrm{e}_{m n}^{B C(k-m)}=I+(B+C) \sum_{i=0, j=0}^{p_{(k)}-1, q_{(k-m)}-1} B^{i} C^{j}\left(\begin{array}{c}
i+j \\
i
\end{array}\right)\left(\begin{array}{c}
k-m-m i-n j \\
i+j+1
\end{array}\right), \\
& \mathrm{e}_{m n}^{B C(k-n)}=I+(B+C) \sum_{i=0, j=0}^{p_{(k-n)}-1, q_{(k)}-2} B^{i} C^{j}\left(\begin{array}{c}
i+j \\
i
\end{array}\right)\left(\begin{array}{c}
k-n-m i-n j \\
i+j+1
\end{array}\right) .
\end{aligned}
$$

Like with the computations performed in the previous part of the proof, we get

$$
\left(\begin{array}{c}
k-m-m i-n j \\
i+j+1
\end{array}\right)=0 \quad \text { if } i \geq 0, j \geq q_{(k-m)}
$$

and

$$
\left(\begin{array}{c}
k-n-m i-n j \\
i+j+1
\end{array}\right)=0 \quad \text { if } i \geq p_{(k-n)}, j \geq 0 .
$$

So, we can substitute $q_{(k-m)}$ by $q_{(k)}$ in (18) and $p_{(k-n)}$ by $p_{(k)}$ in (19).

Accordingly, we have

$$
\mathrm{e}_{m n}^{B C(k-m)}=I+(B+C) \sum_{i=0, j=0}^{p_{(k)}-1, q_{(k)}-1} B^{i} C^{j}\left(\begin{array}{c}
i+j \\
i
\end{array}\right)\left(\begin{array}{c}
k-m(i+1)-n j \\
i+j+1
\end{array}\right),
$$




$$
\mathrm{e}_{m n}^{B C(k-n)}=I+(B+C) \sum_{i=0, j=0}^{p_{(k)}-1, q_{(k)}-2} B^{i} C^{j}\left(\begin{array}{c}
i+j \\
i
\end{array}\right)\left(\begin{array}{c}
k-m i-n(j+1) \\
i+j+1
\end{array}\right) .
$$

It is easy to see that, due to (3), formula (20) can also be used instead of (18) if $q_{(k-m)}<1$ and formula (21) can also be used instead of (19) if $p_{(k-n)}<1$.

We have to prove

$$
\begin{aligned}
\Delta \mathrm{e}_{m n}^{B C k}= & B \mathrm{e}_{m n}^{B C(k-m)}+C \mathrm{e}_{m n}^{B C(k-n)} \\
= & B\left[I+(B+C) \sum_{i=0, j=0}^{p_{(k)}-1, q_{(k)}-1} B^{i} C^{j}\left(\begin{array}{c}
i+j \\
i
\end{array}\right)\left(\begin{array}{c}
k-m(i+1)-n j \\
i+j+1
\end{array}\right)\right] \\
& +C\left[I+(B+C) \sum_{i=0, j=0}^{p_{(k)}-1, q_{(k)}-2} B^{i} C^{j}\left(\begin{array}{c}
i+j \\
i
\end{array}\right)\left(\begin{array}{c}
k-m i-n(j+1) \\
i+j+1
\end{array}\right)\right] .
\end{aligned}
$$

Therefore,

$$
\begin{aligned}
\Delta \mathrm{e}_{m n}^{B C k}= & \mathrm{e}_{m n}^{B C(k+1)}-\mathrm{e}_{m n}^{B C k} \\
= & I+(B+C) \sum_{i=0, j=0}^{p_{(k)}, q_{(k)}-1} B^{i} C^{j}\left(\begin{array}{c}
i+j \\
i
\end{array}\right)\left(\begin{array}{c}
k+1-m i-n j \\
i+j+1
\end{array}\right) \\
& -I-(B+C) \sum_{i=0, j=0}^{p_{(k)}-1, q_{(k)}-1} B^{i} C^{j}\left(\begin{array}{c}
i+j \\
i
\end{array}\right)\left(\begin{array}{c}
k-m i-n j \\
i+j+1
\end{array}\right) \\
= & (B+C)\left[\sum_{i=0, j=0}^{p_{(k)}^{-1, q_{(k)}-1}} B^{i} C^{j}\left(\begin{array}{c}
i+j \\
i
\end{array}\right)\left[\left(\begin{array}{c}
k+1-m i-n j \\
i+j+1
\end{array}\right)-\left(\begin{array}{c}
k-m i-n j \\
i+j+1
\end{array}\right)\right]\right. \\
& \left.+\sum_{j=0}^{q_{(k)}-1} B^{p_{(k)}} C^{j}\left(\begin{array}{c}
p_{(k)}+j \\
p_{(k)}
\end{array}\right)\left(\begin{array}{c}
k+1-m p_{(k)}-n j \\
p_{(k)}+j+1
\end{array}\right)\right] .
\end{aligned}
$$

With the aid of the equation $k=p_{(k)}(m+1)$, we get

$$
\left(\begin{array}{c}
k+1-m p_{(k)}-n j \\
p_{(k)}+j+1
\end{array}\right)=\left(\begin{array}{c}
p_{(k)}+1-n j \\
p_{(k)}+1+j
\end{array}\right)=0 \quad \text { if } j>0
$$

and, by (9), we have

$$
\begin{aligned}
\Delta \mathrm{e}_{m n}^{B C k}= & (B+C)\left[\sum_{i=0, j=0}^{p_{(k)}-1, q_{(k)}-1} B^{i} C^{j}\left(\begin{array}{c}
i+j \\
i
\end{array}\right)\left(\begin{array}{c}
k-m i-n j \\
i+j
\end{array}\right)+B^{p_{(k)}}\right] \\
= & (B+C)\left[I+\sum_{i=1}^{p_{(k)}-1} B^{i} C^{0}\left(\begin{array}{c}
i \\
i
\end{array}\right)\left(\begin{array}{c}
k-m i \\
i
\end{array}\right)+\sum_{j=1}^{q_{(k)}-1} B^{0} C^{j}\left(\begin{array}{c}
j \\
0
\end{array}\right)\left(\begin{array}{c}
k-n j \\
j
\end{array}\right)\right. \\
& \left.+\sum_{i=1, j=1}^{p_{(k)}-1, q_{(k)}-1} B^{i} C^{j}\left(\begin{array}{c}
i+j \\
i
\end{array}\right)\left(\begin{array}{c}
k-m i-n j \\
i+j
\end{array}\right)+B^{p_{(k)}}\right] .
\end{aligned}
$$


By (10), we have

$$
\begin{aligned}
& \Delta \mathrm{e}_{m n}^{B C k}=(B+C)\left[I+\sum_{i=1}^{p_{(k)}-1} B^{i} C^{0}\left(\begin{array}{c}
i-1 \\
i-1
\end{array}\right)\left(\begin{array}{c}
k-m i \\
i
\end{array}\right)+\sum_{j=1}^{q_{(k)}-1} B^{0} C^{j}\left(\begin{array}{c}
j-1 \\
0
\end{array}\right)\left(\begin{array}{c}
k-n j \\
j
\end{array}\right)\right. \\
& +\sum_{i=1, j=1}^{p_{(k)}-1, q_{(k)}-1} B^{i} C^{j}\left(\begin{array}{c}
i+j-1 \\
i-1
\end{array}\right)\left(\begin{array}{c}
k-m i-n j \\
i+j
\end{array}\right) \\
& \left.+\sum_{i=1, j=1}^{p_{(k)}-1, q_{(k)}-1} B^{i} C^{j}\left(\begin{array}{c}
i+j-1 \\
i
\end{array}\right)\left(\begin{array}{c}
k-m i-n j \\
i+j
\end{array}\right)+B^{p_{(k)}}\right] \\
& =(B+C)\left[I+\sum_{i=1, j=0}^{p_{(k)}-1, q_{(k)}-1} B^{i} C^{j}\left(\begin{array}{c}
i+j-1 \\
i-1
\end{array}\right)\left(\begin{array}{c}
k-m i-n j \\
i+j
\end{array}\right)\right. \\
& \left.+\sum_{i=0, j=1}^{p_{(k)}-1, q_{(k)}-1} B^{i} C^{j}\left(\begin{array}{c}
i+j-1 \\
i
\end{array}\right)\left(\begin{array}{c}
k-m i-n j \\
i+j
\end{array}\right)+B^{p_{(k)}}\right] \text {. }
\end{aligned}
$$

Now we replace in the first sum the summation index $i$ by $i+1$ and in the second sum we replace the summation index $j$ by $j+1$. Then

$$
\begin{aligned}
& \Delta \mathrm{e}_{m n}^{B C k}=(B+C)\left[I+\sum_{i=0, j=0}^{p_{(k)}-2, q_{(k)}-1} B^{i+1} C^{j}\left(\begin{array}{c}
i+j \\
i
\end{array}\right)\left(\begin{array}{c}
k-m(i+1)-n j \\
i+j+1
\end{array}\right)\right. \\
& \left.+\sum_{i=0, j=0}^{p_{(k)}-1, q_{(k)}-2} B^{i} C^{j+1}\left(\begin{array}{c}
i+j \\
i
\end{array}\right)\left(\begin{array}{c}
k-m i-n(j+1) \\
i+j+1
\end{array}\right)+B^{p_{(k)}}\right] \\
& =B+B(B+C) \sum_{i=0, j=0}^{p_{(k)}-2, q_{(k)}-1} B^{i} C^{j}\left(\begin{array}{c}
i+j \\
i
\end{array}\right)\left(\begin{array}{c}
k-m(i+1)-n j \\
i+j+1
\end{array}\right)+B^{p_{(k)}}(B+C) \\
& +C+C(B+C) \sum_{i=0, j=0}^{p_{(k)}-1, q_{(k)}-2} B^{i} C^{j}\left(\begin{array}{c}
i+j \\
i
\end{array}\right)\left(\begin{array}{c}
k-m i-n(j+1) \\
i+j+1
\end{array}\right) \\
& =B\left[I+(B+C)\left(\sum_{i=0, j=0}^{p_{(k)}-2, q_{(k)}-1} B^{i} C^{j}\left(\begin{array}{c}
i+j \\
i
\end{array}\right)\left(\begin{array}{c}
k-m(i+1)-n j \\
i+j+1
\end{array}\right)+B^{p_{(k)}-1}\right)\right] \\
& +C\left[I+(B+C) \sum_{i=0, j=0}^{p_{(k)}-1, q_{(k)}-2} B^{i} C^{j}\left(\begin{array}{c}
i+j \\
i
\end{array}\right)\left(\begin{array}{c}
k-m i-n(j+1) \\
i+j+1
\end{array}\right)\right] .
\end{aligned}
$$

For $k=p_{(k)}(m+1)$, we have

$$
B^{p_{(k)}-1}=\sum_{j=0}^{q_{(k)}-1} B^{p_{(k)}-1} C^{j}\left(\begin{array}{c}
p_{(k)}-1+j \\
p_{(k)}-1
\end{array}\right)\left(\begin{array}{c}
k-m\left(p_{(k)}-1+1\right)-n j \\
p_{(k)}-1+j+1
\end{array}\right),
$$

where

$$
\left(\begin{array}{c}
k-m\left(p_{(k)}-1+1\right)-n j \\
p_{(k)}-1+j+1
\end{array}\right)=\left(\begin{array}{c}
k-m p_{(k)}-n j \\
p_{(k)}+j
\end{array}\right)=0 \quad \text { if } j>0 .
$$


Thus,

$$
\begin{aligned}
\Delta \mathrm{e}_{m n}^{B C k}= & B\left[I+(B+C) \sum_{i=0, j=0}^{p_{(k)}-1, q_{(k)}-1} B^{i} C^{j}\left(\begin{array}{c}
i+j \\
i
\end{array}\right)\left(\begin{array}{c}
k-m(i+1)-n j \\
i+j+1
\end{array}\right)\right] \\
& +C\left[I+(B+C) \sum_{i=0, j=0}^{p_{(k)}-1, q_{(k)}-2} B^{i} C^{j}\left(\begin{array}{c}
i+j \\
i
\end{array}\right)\left(\begin{array}{c}
k-m i-n(j+1) \\
i+j+1
\end{array}\right)\right] \\
= & B \mathrm{e}_{m n}^{B C(k-m)}+C \mathrm{e}_{m n}^{B C(k-n)}
\end{aligned}
$$

and formula (22) is proved.

III. $\left(p_{(k)}-1\right)(m+1)+1 \leq k<p_{(k)}(m+1) \wedge k=q_{(k)}(n+1)$

In this case, we have (see relevant computations in cases I and II)

$$
p_{(k-m)}=p_{(k)}-1, \quad p_{(k+1)}=p_{(k)}
$$

and

$$
q_{(k-n)}=q_{(k)}, \quad q_{(k+1)}=q_{(k)}+1 .
$$

Then

$$
\begin{aligned}
& \mathrm{e}_{m n}^{B C k}=I+(B+C) \sum_{i=0, j=0}^{p_{(k)}-1, q_{(k)}-1} B^{i} C^{j}\left(\begin{array}{c}
i+j \\
i
\end{array}\right)\left(\begin{array}{c}
k-m i-n j \\
i+j+1
\end{array}\right), \\
& \mathrm{e}_{m n}^{B C(k+1)}=I+(B+C) \sum_{i=0, j=0}^{p_{(k)}-1, q_{(k)}} B^{i} C^{j}\left(\begin{array}{c}
i+j \\
i
\end{array}\right)\left(\begin{array}{c}
k+1-m i-n j \\
i+j+1
\end{array}\right)
\end{aligned}
$$

and

$$
\begin{aligned}
& \mathrm{e}_{m n}^{B C(k-m)}=I+(B+C) \sum_{i=0, j=0}^{p_{(k)}-2, q_{(k-m)}-1} B^{i} C^{j}\left(\begin{array}{c}
i+j \\
i
\end{array}\right)\left(\begin{array}{c}
k-m-m i-n j \\
i+j+1
\end{array}\right), \\
& \mathrm{e}_{m n}^{B C(k-n)}=I+(B+C) \sum_{i=0, j=0}^{p_{(k-n)}-1, q_{(k)}-1} B^{i} C^{j}\left(\begin{array}{c}
i+j \\
i
\end{array}\right)\left(\begin{array}{c}
k-n-m i-n j \\
i+j+1
\end{array}\right) .
\end{aligned}
$$

Like with the computations performed in case I, we can get

$$
\left(\begin{array}{c}
k-m-m i-n j \\
i+j+1
\end{array}\right)=0 \quad \text { if } i \geq 0, j \geq q_{(k-m)}
$$

and

$$
\left(\begin{array}{c}
k-n-m i-n j \\
i+j+1
\end{array}\right)=0 \quad \text { if } i \geq p_{(k-n)}, j \geq 0 .
$$

So, we can substitute $q_{(k)}$ for $q_{(k-m)}$ in (23) and $p_{(k)}$ for $p_{(k-n)}$ in (24). 
Thus, we have

$$
\begin{aligned}
& \mathrm{e}_{m n}^{B C(k-m)}=I+(B+C) \sum_{i=0, j=0}^{p_{(k)}-2, q_{(k)}-1} B^{i} C^{j}\left(\begin{array}{c}
i+j \\
i
\end{array}\right)\left(\begin{array}{c}
k-m(i+1)-n j \\
i+j+1
\end{array}\right), \\
& \mathrm{e}_{m n}^{B C(k-n)}=I+(B+C) \sum_{i=0, j=0}^{p_{(k)}-1, q_{(k)}-1} B^{i} C^{j}\left(\begin{array}{c}
i+j \\
i
\end{array}\right)\left(\begin{array}{c}
k-m i-n(j+1) \\
i+j+1
\end{array}\right) .
\end{aligned}
$$

It is easy to see that, due to (3), formula (25) can also be used instead of (23) if $q_{(k-m)}<1$ and formula (26) can also be used instead of (24) if $p_{(k-n)}<1$.

Now we have to prove

$$
\begin{aligned}
\Delta \mathrm{e}_{m n}^{B C k}= & B \mathrm{e}_{m n}^{B C(k-m)}+C \mathrm{e}_{m n}^{B C(k-n)} \\
= & B\left[I+(B+C) \sum_{i=0, j=0}^{p_{(k)}-2, q_{(k)}-1} B^{i} C^{j}\left(\begin{array}{c}
i+j \\
i
\end{array}\right)\left(\begin{array}{c}
k-m(i+1)-n j \\
i+j+1
\end{array}\right)\right] \\
& +C\left[I+(B+C) \sum_{i=0, j=0}^{p_{(k)}-1, q_{(k)}-1} B^{i} C^{j}\left(\begin{array}{c}
i+j \\
i
\end{array}\right)\left(\begin{array}{c}
k-m i-n(j+1) \\
i+j+1
\end{array}\right)\right] .
\end{aligned}
$$

Considering the difference by its definition, we get

$$
\begin{aligned}
\Delta \mathrm{e}_{m n}^{B C k}= & \mathrm{e}_{m n}^{B C(k+1)}-\mathrm{e}_{m n}^{B C k} \\
= & I+(B+C) \sum_{i=0, j=0}^{p_{(k)}-1, q_{(k)}} B^{i} C^{j}\left(\begin{array}{c}
i+j \\
i
\end{array}\right)\left(\begin{array}{c}
k+1-m i-n j \\
i+j+1
\end{array}\right) \\
& -I-(B+C) \sum_{i=0, j=0}^{p_{(k)}-1, q_{(k)}-1} B^{i} C^{j}\left(\begin{array}{c}
i+j \\
i
\end{array}\right)\left(\begin{array}{c}
k-m i-n j \\
i+j+1
\end{array}\right) \\
= & (B+C)\left[\sum_{i=0, j=0}^{p_{(k)}^{-1, q_{(k)}-1}} B^{i} C^{j}\left(\begin{array}{c}
i+j \\
i
\end{array}\right)\left[\left(\begin{array}{c}
k+1-m i-n j \\
i+j+1
\end{array}\right)-\left(\begin{array}{c}
k-m i-n j \\
i+j+1
\end{array}\right)\right]\right. \\
& \left.+\sum_{i=0}^{p_{(k)}-1} B^{i} C^{q_{(k)}}\left(\begin{array}{c}
i+q_{(k)} \\
i
\end{array}\right)\left(\begin{array}{c}
k+1-m i-n q(k) \\
i+q_{(k)}+1
\end{array}\right)\right]
\end{aligned}
$$

With the aid of relation $k=q_{(k)}(n+1)$, we get

$$
\left(\begin{array}{c}
k+1-m i-n q_{(k)} \\
i+q_{(k)}+1
\end{array}\right)=\left(\begin{array}{c}
q_{(k)}+1-m i \\
q_{(k)}+1+i
\end{array}\right)=0 \quad \text { if } i>0
$$

and

$$
\begin{aligned}
\Delta \mathrm{e}_{m n}^{B C k} & =(B+C)\left[\sum_{i=0, j=0}^{p_{(k)}-1, q_{(k)}-1} B^{i} C^{j}\left(\begin{array}{c}
i+j \\
i
\end{array}\right)\left(\begin{array}{c}
k-m i-n j \\
i+j
\end{array}\right)+C^{q_{(k)}}\right] \\
& =(B+C)\left[I+\sum_{i=1}^{p_{(k)}-1} B^{i} C^{0}\left(\begin{array}{c}
i \\
i
\end{array}\right)\left(\begin{array}{c}
k-m i \\
i
\end{array}\right)\right.
\end{aligned}
$$




$$
\begin{aligned}
& +\sum_{j=1}^{q_{(k)}-1} B^{0} C^{j}\left(\begin{array}{l}
j \\
0
\end{array}\right)\left(\begin{array}{c}
k-n j \\
j
\end{array}\right) \\
& \left.+\sum_{i=1, j=1}^{p_{(k)}-1, q_{(k)}-1} B^{i} C^{j}\left(\begin{array}{c}
i+j \\
i
\end{array}\right)\left(\begin{array}{c}
k-m i-n j \\
i+j
\end{array}\right)+C^{q_{(k)}}\right] .
\end{aligned}
$$

By (10), we have

$$
\begin{aligned}
& \Delta \mathrm{e}_{m n}^{B C k}=(B+C)\left[I+\sum_{i=1}^{p_{(k)}-1} B^{i} C^{0}\left(\begin{array}{c}
i-1 \\
i-1
\end{array}\right)\left(\begin{array}{c}
k-m i \\
i
\end{array}\right)+\sum_{j=1}^{q_{(k)}-1} B^{0} C^{j}\left(\begin{array}{c}
j-1 \\
0
\end{array}\right)\left(\begin{array}{c}
k-n j \\
j
\end{array}\right)\right. \\
& +\sum_{i=1, j=1}^{p_{(k)}-1, q_{(k)}-1} B^{i} C^{j}\left(\begin{array}{c}
i+j-1 \\
i-1
\end{array}\right)\left(\begin{array}{c}
k-m i-n j \\
i+j
\end{array}\right) \\
& \left.+\sum_{i=1, j=1}^{p_{(k)}-1, q_{(k)}-1} B^{i} C^{j}\left(\begin{array}{c}
i+j-1 \\
i
\end{array}\right)\left(\begin{array}{c}
k-m i-n j \\
i+j
\end{array}\right)+C^{q_{(k)}}\right] \\
& =(B+C)\left[I+\sum_{i=1, j=0}^{p_{(k)}-1, q_{(k)}-1} B^{i} C^{j}\left(\begin{array}{c}
i+j-1 \\
i-1
\end{array}\right)\left(\begin{array}{c}
k-m i-n j \\
i+j
\end{array}\right)\right. \\
& \left.+\sum_{i=0, j=1}^{p_{(k)}-1, q_{(k)}-1} B^{i} C^{j}\left(\begin{array}{c}
i+j-1 \\
i
\end{array}\right)\left(\begin{array}{c}
k-m i-n j \\
i+j
\end{array}\right)+C^{q_{(k)}}\right] \text {. }
\end{aligned}
$$

Now we replace in the first sum the summation index $i$ by $i+1$ and in the second sum we replace the summation index $j$ by $j+1$. Then

$$
\begin{aligned}
\Delta \mathrm{e}_{m n}^{B C k}= & (B+C)\left[I+\sum_{i=0, j=0}^{p(k)-2, q_{(k)}-1} B^{i+1} C^{j}\left(\begin{array}{c}
i+j \\
i
\end{array}\right)\left(\begin{array}{c}
k-m(i+1)-n j \\
i+j+1
\end{array}\right)\right. \\
& \left.+\sum_{i=0, j=0}^{p(k)-1, q_{(k)}-2} B^{i} C^{j+1}\left(\begin{array}{c}
i+j \\
i
\end{array}\right)\left(\begin{array}{c}
k-m i-n(j+1) \\
i+j+1
\end{array}\right)+C^{q(k)}\right] \\
= & B+B(B+C) \sum_{i=0, j=0}^{p_{(k)}-2, q_{(k)}-1} B^{i} C^{j}\left(\begin{array}{c}
i+j \\
i
\end{array}\right)\left(\begin{array}{c}
k-m(i+1)-n j \\
i+j+1
\end{array}\right) \\
& +C+C(B+C) \sum_{i=0, j=0}^{p_{(k)}-1, q_{(k)}-2} B^{i} C^{j}\left(\begin{array}{c}
i+j \\
i
\end{array}\right)\left(\begin{array}{c}
k-m i-n(j+1) \\
i+j+1
\end{array}\right)+C^{q_{(k)}(B+C)}(B) \\
= & B\left[I+(B+C) \sum_{i=0, j=0}^{p_{(k)}-2, q_{(k)}-1} B^{i} C^{j}\left(\begin{array}{c}
i+j \\
i
\end{array}\right)\left(\begin{array}{c}
k-m(i+1)-n j \\
i+j+1
\end{array}\right)\right] \\
& +C\left[I+(B+C)\left(\sum_{i=0, j=0}^{p_{(k)}-1, q_{(k)}-2} B^{i} C^{j}\left(\begin{array}{c}
i+j \\
i
\end{array}\right)\left(\begin{array}{c}
k-m i-n(j+1) \\
i+j+1
\end{array}\right)+C^{q_{(k)}-1}\right)\right] .
\end{aligned}
$$

For $k=q_{(k)}(n+1)$, we have

$$
C^{q_{(k)}-1}=\sum_{i=0}^{p_{(k)}-1} B^{i} C^{q_{(k)}-1}\left(\begin{array}{c}
i+q_{(k)}-1 \\
i
\end{array}\right)\left(\begin{array}{c}
k-m i-n\left(q_{(k)}-1+1\right) \\
i+q_{(k)}-1+1
\end{array}\right),
$$


where

$$
\left(\begin{array}{c}
k-m i-n\left(q_{(k)}-1+1\right) \\
i+q_{(k)}-1+1
\end{array}\right)=\left(\begin{array}{c}
k-m i-n q_{(k)} \\
i+q_{(k)}
\end{array}\right)=0 \quad \text { if } i>0 .
$$

Thus,

$$
\begin{aligned}
\Delta \mathrm{e}_{m n}^{B C k}= & B\left[I+(B+C) \sum_{i=0, j=0}^{p_{(k)}-2, q_{(k)}-1} B^{i} C^{j}\left(\begin{array}{c}
i+j \\
i
\end{array}\right)\left(\begin{array}{c}
k-m(i+1)-n j \\
i+j+1
\end{array}\right)\right] \\
& +C\left[I+(B+C) \sum_{i=0, j=0}^{p_{(k)}-1, q_{(k)}-1} B^{i} C^{j}\left(\begin{array}{c}
i+j \\
i
\end{array}\right)\left(\begin{array}{c}
k-m i-n(j+1) \\
i+j+1
\end{array}\right)\right] \\
= & B \mathrm{e}_{m n}^{B C(k-m)}+C \mathrm{e}_{m n}^{B C(k-n)}
\end{aligned}
$$

and formula (27) is proved.

IV. $k=p_{(k)}(m+1) \wedge k=q_{(k)}(n+1)$

In this case, we have (see similar combinations in cases II and III)

$$
p_{(k-m)}=p_{(k)}, \quad p_{(k+1)}=p_{(k)}+1
$$

and

$$
q_{(k-n)}=q_{(k)}, \quad q_{(k+1)}=q_{(k)}+1 .
$$

Then

$$
\begin{aligned}
& \mathrm{e}_{m n}^{B C k}=I+(B+C) \sum_{i=0, j=0}^{p_{(k)}-1, q_{(k)}-1} B^{i} C^{j}\left(\begin{array}{c}
i+j \\
i
\end{array}\right)\left(\begin{array}{c}
k-m i-n j \\
i+j+1
\end{array}\right), \\
& \mathrm{e}_{m n}^{B C(k+1)}=I+(B+C) \sum_{i=0, j=0}^{p_{(k)}, q_{(k)}} B^{i} C^{j}\left(\begin{array}{c}
i+j \\
i
\end{array}\right)\left(\begin{array}{c}
k+1-m i-n j \\
i+j+1
\end{array}\right)
\end{aligned}
$$

and

$$
\begin{aligned}
& \mathrm{e}_{m n}^{B C(k-m)}=I+(B+C) \sum_{i=0, j=0}^{p_{(k)}-1, q_{(k-m)}-1} B^{i} C^{j}\left(\begin{array}{c}
i+j \\
i
\end{array}\right)\left(\begin{array}{c}
k-m-m i-n j \\
i+j+1
\end{array}\right), \\
& \mathrm{e}_{m n}^{B C(k-n)}=I+(B+C) \sum_{i=0, j=0}^{p_{(k-n)}-1, q_{(k)}-1} B^{i} C^{j}\left(\begin{array}{c}
i+j \\
i
\end{array}\right)\left(\begin{array}{c}
k-n-m i-n j \\
i+j+1
\end{array}\right) .
\end{aligned}
$$

As before,

$$
\left(\begin{array}{c}
k-m-m i-n j \\
i+j+1
\end{array}\right)=0 \quad \text { if } i \geq 0, j \geq q_{(k-m)}
$$

and

$$
\left(\begin{array}{c}
k-n-m i-n j \\
i+j+1
\end{array}\right)=0 \quad \text { if } i \geq p_{(k-n)}, j \geq 0 .
$$


So, we can substitute $q_{(k)}$ for $q_{(k-m)}$ in (28) and $p_{(k)}$ for $p_{(k-n)}$ in (29) and

$$
\begin{aligned}
& \mathrm{e}_{m n}^{B C(k-m)}=I+(B+C) \sum_{i=0, j=0}^{p_{(k)}-1, q_{(k)}-1} B^{i} C^{j}\left(\begin{array}{c}
i+j \\
i
\end{array}\right)\left(\begin{array}{c}
k-m(i+1)-n j \\
i+j+1
\end{array}\right), \\
& \mathrm{e}_{m n}^{B C(k-n)}=I+(B+C) \sum_{i=0, j=0}^{p_{(k)}-1, q_{(k)}-1} B^{i} C^{j}\left(\begin{array}{c}
i+j \\
i
\end{array}\right)\left(\begin{array}{c}
k-m i-n(j+1) \\
i+j+1
\end{array}\right) .
\end{aligned}
$$

It is easy to see that, due to (3), formula (30) can also be used instead of (28) if $q_{(k-m)}<1$ and formula (31) can also be used instead of $(29)$ if $p_{(k-n)}<1$.

Now it is possible to prove the formula

$$
\begin{aligned}
\Delta \mathrm{e}_{m n}^{B C k}= & B \mathrm{e}_{m n}^{B C(k-m)}+C \mathrm{e}_{m n}^{B C(k-n)} \\
= & B\left[I+(B+C) \sum_{i=0, j=0}^{p_{(k)}-1, q_{(k)}-1} B^{i} C^{j}\left(\begin{array}{c}
i+j \\
i
\end{array}\right)\left(\begin{array}{c}
k-m(i+1)-n j \\
i+j+1
\end{array}\right)\right] \\
& +C\left[I+(B+C) \sum_{i=0, j=0}^{p_{(k)}-1, q_{(k)}-1} B^{i} C^{j}\left(\begin{array}{c}
i+j \\
i
\end{array}\right)\left(\begin{array}{c}
k-m i-n(j+1) \\
i+j+1
\end{array}\right)\right] .
\end{aligned}
$$

By definition, we get

$$
\begin{aligned}
& \Delta \mathrm{e}_{m n}^{B C k}=\mathrm{e}_{m n}^{B C(k+1)}-\mathrm{e}_{m n}^{B C k} \\
& =I+(B+C) \sum_{i=0, j=0}^{p_{(k)}, q_{(k)}} B^{i} C^{j}\left(\begin{array}{c}
i+j \\
i
\end{array}\right)\left(\begin{array}{c}
k+1-m i-n j \\
i+j+1
\end{array}\right) \\
& -I-(B+C) \sum_{i=0, j=0}^{p_{(k)}-1, q_{(k)}-1} B^{i} C^{j}\left(\begin{array}{c}
i+j \\
i
\end{array}\right)\left(\begin{array}{c}
k-m i-n j \\
i+j+1
\end{array}\right) \\
& =(B+C)\left[\sum_{i=0, j=0}^{p_{(k)}-1, q_{(k)}-1} B^{i} C^{j}\left(\begin{array}{c}
i+j \\
i
\end{array}\right)\left[\left(\begin{array}{c}
k+1-m i-n j \\
i+j+1
\end{array}\right)-\left(\begin{array}{c}
k-m i-n j \\
i+j+1
\end{array}\right)\right]\right. \\
& +\sum_{j=0}^{q_{(k)}} B^{p_{(k)}} C^{j}\left(\begin{array}{c}
p_{(k)}+j \\
p_{(k)}
\end{array}\right)\left(\begin{array}{c}
k+1-m p_{(k)}-n j \\
p_{(k)}+j+1
\end{array}\right) \\
& \left.+\sum_{i=0}^{p_{(k)}} B^{i} C^{q_{(k)}}\left(\begin{array}{c}
i+q_{(k)} \\
i
\end{array}\right)\left(\begin{array}{c}
k+1-m i-n q_{(k)} \\
i+q_{(k)}+1
\end{array}\right)\right] .
\end{aligned}
$$

With the aid of equations $k=p_{(k)}(m+1), k=q_{(k)}(n+1)$, we get

$$
\begin{aligned}
& \left(\begin{array}{c}
k+1-m p_{(k)}-n j \\
p_{(k)}+j+1
\end{array}\right)=\left(\begin{array}{c}
p_{(k)}+1-n j \\
p_{(k)}+1+j
\end{array}\right)=0 \quad \text { if } j>0, \\
& \left(\begin{array}{c}
k+1-m i-n q_{(k)} \\
i+q_{(k)}+1
\end{array}\right)=\left(\begin{array}{c}
q_{(k)}+1-m i \\
q_{(k)}+1+i
\end{array}\right)=0 \quad \text { if } i>0
\end{aligned}
$$


and

$$
\begin{aligned}
\Delta \mathrm{e}_{m n}^{B C k}= & (B+C)\left[\sum_{i=0, j=0}^{p_{(k)}-1, q_{(k)}-1} B^{i} C^{j}\left(\begin{array}{c}
i+j \\
i
\end{array}\right)\left(\begin{array}{c}
k-m i-n j \\
i+j
\end{array}\right)+B^{p_{(k)}}+C^{q_{(k)}}\right] \\
= & (B+C)\left[I+\sum_{i=1}^{p_{(k)}-1} B^{i} C^{0}\left(\begin{array}{c}
i \\
i
\end{array}\right)\left(\begin{array}{c}
k-m i \\
i
\end{array}\right)+\sum_{j=1}^{q_{(k)}-1} B^{0} C^{j}\left(\begin{array}{c}
j \\
0
\end{array}\right)\left(\begin{array}{c}
k-n j \\
j
\end{array}\right)\right. \\
& \left.+\sum_{i=1, j=1}^{p_{(k)}-1, q_{(k)}-1} B^{i} C^{j}\left(\begin{array}{c}
i+j \\
i
\end{array}\right)\left(\begin{array}{c}
k-m i-n j \\
i+j
\end{array}\right)+B^{p_{(k)}}+C^{q_{(k)}}\right] .
\end{aligned}
$$

By (10), we have

$$
\begin{aligned}
& \Delta \mathrm{e}_{m n}^{B C k}=(B+C)\left[I+\sum_{i=1}^{p_{(k)}-1} B^{i} C^{0}\left(\begin{array}{c}
i-1 \\
i-1
\end{array}\right)\left(\begin{array}{c}
k-m i \\
i
\end{array}\right)+\sum_{j=1}^{q_{(k)}-1} B^{0} C^{j}\left(\begin{array}{c}
j-1 \\
0
\end{array}\right)\left(\begin{array}{c}
k-n j \\
j
\end{array}\right)\right. \\
& +\sum_{i=1, j=1}^{p_{(k)}-1, q_{(k)}-1} B^{i} C^{j}\left(\begin{array}{c}
i+j-1 \\
i-1
\end{array}\right)\left(\begin{array}{c}
k-m i-n j \\
i+j
\end{array}\right) \\
& \left.+\sum_{i=1, j=1}^{p_{(k)}-1, q_{(k)}-1} B^{i} C^{j}\left(\begin{array}{c}
i+j-1 \\
i
\end{array}\right)\left(\begin{array}{c}
k-m i-n j \\
i+j
\end{array}\right)+B^{p_{(k)}}+C^{q_{(k)}}\right] \\
& =(B+C)\left[I+\sum_{i=1, j=0}^{p_{(k)}-1, q_{(k)}-1} B^{i} C^{j}\left(\begin{array}{c}
i+j-1 \\
i-1
\end{array}\right)\left(\begin{array}{c}
k-m i-n j \\
i+j
\end{array}\right)\right. \\
& \left.+\sum_{i=0, j=1}^{p_{(k)}-1, q_{(k)}-1} B^{i} C^{j}\left(\begin{array}{c}
i+j-1 \\
i
\end{array}\right)\left(\begin{array}{c}
k-m i-n j \\
i+j
\end{array}\right)+B^{p_{(k)}}+C^{q_{(k)}}\right] .
\end{aligned}
$$

We replace in the first sum the summation index $i$ by $i+1$ and in the second sum we substitute the summation index $j$ by $j+1$. Then

$$
\begin{aligned}
\Delta \mathrm{e}_{m n}^{B C k}= & (B+C)\left[I+\sum_{i=0, j=0}^{p_{(k)}-2, q_{(k)}-1} B^{i+1} C^{j}\left(\begin{array}{c}
i+j \\
i
\end{array}\right)\left(\begin{array}{c}
k-m(i+1)-n j \\
i+j+1
\end{array}\right)\right. \\
& \left.+\sum_{i=0, j=0}^{p_{(k)}-1, q_{(k)}-2} B^{i} C^{j+1}\left(\begin{array}{c}
i+j \\
i
\end{array}\right)\left(\begin{array}{c}
k-m i-n(j+1) \\
i+j+1
\end{array}\right)+B^{p_{(k)}+C^{q_{(k)}}}\right] \\
= & B+B(B+C) \sum_{i=0, j=0}^{p_{(k)}-2, q_{(k)}-1} B^{i} C^{j}\left(\begin{array}{c}
i+j \\
i
\end{array}\right)\left(\begin{array}{c}
k-m(i+1)-n j \\
i+j+1
\end{array}\right)+B^{p_{(k)}(B+C)} \\
& +C+C(B+C) \sum_{i=0, j=0}^{p_{(k)}-1, q_{(k)}-2} B^{i} C^{j}\left(\begin{array}{c}
i+j \\
i
\end{array}\right)\left(\begin{array}{c}
k-m i-n(j+1) \\
i+j+1
\end{array}\right)+C^{q_{(k)}(B+C)} \\
= & B\left[I+(B+C)\left(\sum_{i=0, j=0}^{p_{(k)}-2, q_{(k)}-1} B^{i} C^{j}\left(\begin{array}{c}
i+j \\
i
\end{array}\right)\left(\begin{array}{c}
k-m(i+1)-n j \\
i+j+1
\end{array}\right)+B^{p_{(k)}-1}\right)\right] \\
& +C\left[I+(B+C)\left(\sum_{i=0, j=0}^{p_{(k)}-1, q_{(k)}-2} B^{i} C^{j}\left(\begin{array}{c}
i+j \\
i
\end{array}\right)\left(\begin{array}{c}
k-m i-n(j+1) \\
i+j+1
\end{array}\right)+C^{q_{(k)}-1}\right)\right] .
\end{aligned}
$$


Because $k=p_{(k)}(m+1)=q_{(k)}(n+1)$, we can express $B^{p_{(k)}-1}$ and $C^{q_{(k)}-1}$ in the form

$$
\begin{aligned}
& B^{p_{(k)}-1}=\sum_{j=0}^{q_{(k)}-1} B^{p_{(k)}-1} C^{j}\left(\begin{array}{c}
p_{(k)}-1+j \\
p_{(k)}-1
\end{array}\right)\left(\begin{array}{c}
k-m\left(p_{(k)}-1+1\right)-n j \\
p_{(k)}-1+j+1
\end{array}\right), \\
& C^{q_{(k)}-1}=\sum_{i=0}^{p_{(k)}-1} B^{i} C^{q_{(k)}-1}\left(\begin{array}{c}
i+q_{(k)}-1 \\
i
\end{array}\right)\left(\begin{array}{c}
k-m i-n\left(q_{(k)}-1+1\right) \\
i+q_{(k)}-1+1
\end{array}\right),
\end{aligned}
$$

where

$$
\begin{aligned}
& \left(\begin{array}{c}
k-m\left(p_{(k)}-1+1\right)-n j \\
p_{(k)}-1+j+1
\end{array}\right)=\left(\begin{array}{c}
k-m p_{(k)}-n j \\
p_{(k)}+j
\end{array}\right)=0 \quad \text { if } j>0, \\
& \left(\begin{array}{c}
k-m i-n\left(q_{(k)}-1+1\right) \\
i+q_{(k)}-1+1
\end{array}\right)=\left(\begin{array}{c}
k-m i-n q_{(k)} \\
i+q_{(k)}
\end{array}\right)=0 \quad \text { if } i>0 .
\end{aligned}
$$

Thus,

$$
\begin{aligned}
\Delta \mathrm{e}_{m n}^{B C k}= & B\left[I+(B+C) \sum_{i=0, j=0}^{p_{(k)}^{-1, q_{(k)}-1}} B^{i} C^{j}\left(\begin{array}{c}
i+j \\
i
\end{array}\right)\left(\begin{array}{c}
k-m(i+1)-n j \\
i+j+1
\end{array}\right)\right] \\
& +C\left[I+(B+C) \sum_{i=0, j=0}^{p_{(k)}-1, q_{(k)}-1} B^{i} C^{j}\left(\begin{array}{c}
i+j \\
i
\end{array}\right)\left(\begin{array}{c}
k-m i-n(j+1) \\
i+j+1
\end{array}\right)\right] \\
= & B \mathrm{e}_{m n}^{B C(k-m)}+C \mathrm{e}_{m n}^{B C(k-n)} .
\end{aligned}
$$

Therefore, formula (32) is valid.

We proved that formula (6) holds in each of the considered cases I, II, III and IV for $k \geq 1$. If $k=0$, the proof can be done directly because $p_{(0)}=q_{(0)}=0, p_{(1)}=q_{(1)}=1$,

$$
\begin{aligned}
\Delta \mathrm{e}_{m n}^{B C 0}= & \mathrm{e}_{m n}^{B C 1}-\mathrm{e}_{m n}^{B C 0} \\
= & I+(B+C) \sum_{i=0, j=0}^{0,0} B^{i} C^{j}\left(\begin{array}{c}
i+j \\
i
\end{array}\right)\left(\begin{array}{c}
1-m i-n j \\
i+j+1
\end{array}\right) \\
& -I-(B+C) \sum_{i=0, j=0}^{-1,-1} B^{i} C^{j}\left(\begin{array}{c}
i+j \\
i
\end{array}\right)\left(\begin{array}{c}
-m i-n j \\
i+j+1
\end{array}\right)=I+B+C-I=B+C
\end{aligned}
$$

and

$$
B \mathrm{e}_{m n}^{B C(-m)}+C \mathrm{e}_{m n}^{B C(-n)}=B I+C I=B+C .
$$

Formula (6) holds again. Theorem 2 is proved.

\section{Open problems and concluding remarks}

Formula (4) is valid for $k \in \mathbb{Z}_{-m}^{\infty}$. However, formula (6) holds for $k \in \mathbb{Z}_{0}^{\infty}$ only. Therefore, there is a difference between the definition domains of the formulas, and it is a challenge how to modify Definition 2 of discrete matrix delayed exponential for two delays in such 
a way that formula (6) will hold for $k \in \mathbb{Z}_{-\max \{m, n\}}^{\infty}$. In [1] formula (4) is used to get a representation of the solution of the problems (both homogeneous and nonhomogeneous)

$$
\begin{aligned}
& \Delta y(k)=B y(k-m)+f(k), \quad k \in \mathbb{Z}_{0}^{\infty}, \\
& y(k)=\varphi(k), \quad k \in \mathbb{Z}_{-m}^{0},
\end{aligned}
$$

where $f: \mathbb{Z}_{0}^{\infty} \rightarrow \mathbb{R}^{r}, y: \mathbb{Z}_{-m}^{\infty} \rightarrow \mathbb{R}^{r}$ and $\varphi: \mathbb{Z}_{-m}^{0} \rightarrow \mathbb{R}^{r}$.

It is an open problem how to use formula (6) to get a representation of the solution of the homogeneous and nonhomogeneous problems

$$
\begin{aligned}
& \Delta y(k)=B y(k-m)+C y(k-n)+f(k), \quad k \in \mathbb{Z}_{0}^{\infty}, \\
& y(k)=\varphi(k), \quad k \in \mathbb{Z}_{-s}^{0}, s=\max \{m, n\}
\end{aligned}
$$

if $B C=C B$.

Let us note that the first concept of matrix delayed exponential was given in [3] and the first concept of discrete matrix delayed exponential was given in [1]. Further development of the delayed matrix exponentials method and its utilization to various problems can be found, e.g., in [4-16].

\section{Competing interests}

The authors declare that they have no competing interests.

\section{Authors' contributions}

The authors have made the same contribution. Both authors have read and approved the final manuscript.

\section{Author details}

'Department of Mathematics and Descriptive Geometry, AdMaS, Faculty of Civil Engineering, Brno University of Technology, Brno, Czech Republic. ${ }^{2}$ Department of Mathematics, Faculty of Electrical Engineering and Communication, Brno University of Technology, Brno, Czech Republic. ${ }^{3}$ Department of Mathematics and Descriptive Geometry, Faculty of Civil Engineering, Brno University of Technology, Brno, Czech Republic.

\section{Acknowledgements}

The first author was supported by Operational Programme Research and Development for Innovations, No. CZ.1.05/2.1.00/03.0097, as an activity of the regional Centre AdMaS.

Received: 23 January 2013 Accepted: 29 April 2013 Published: 14 May 2013

\section{References}

1. Diblík, J, Khusainov, DY: Representation of solutions of linear discrete systems with constant coefficients and pure delay. Adv. Differ. Equ. 2006, Article ID 80825 (2006)

2. Diblík, J, Khusainov, DY: Representation of solutions of discrete delayed system $x(k+1)=A x(k)+B x(k-m)+f(k)$ with commutative matrices. J. Math. Anal. Appl. 318(1), 63-76 (2006)

3. Khusainov, DY, Shuklin, GV: Linear autonomous time-delay system with permutation matrices solving. Stud. Univ. Žilina, Math. Ser. 17, 101-108 (2003)

4. Boichuk, A, Diblík, J, Khusainov, D, Rưžičková, M: Boundary value problems for delay differential systems. Adv. Differ. Equ. 2010, Article ID 593834 (2010)

5. Boichuk, A, Diblík, J, Khusainov, D, Růžičková, M: Fredholm's boundary-value problems for differential systems with a single delay. Nonlinear Anal., Theory Methods Appl. 72, 2251-2258 (2010)

6. Boichuk, A, Diblík, J, Khusainov, D, Růžičková, M: Boundary-value problems for weakly nonlinear delay differential systems. Abstr. Appl. Anal. 2011, Article ID 631412 (2011)

7. Diblík, J, Fečkan, M, Pospiśsil, M: Representation of a solution of the Cauchy problem for an oscillating system with two delays and permutable matrices. Ukr. Mat. Zh. 65(1), 58-69 (2013)

8. Diblík, J, Khusainov, D, Kukharenko, O, Svoboda, Z: Solution of the first boundary-value problem for a system of autonomous second-order linear PDEs of parabolic type with a single delay. Abstr. Appl. Anal. 2012, Article ID 219040 (2012)

9. Diblík, J, Khusainov, DY, Lukáčová, J, Rưžičková, M: Control of oscillating systems with a single delay. Adv. Differ. Equ. 2010, Article ID 108218 (2010)

10. Diblík, J, Khusainov, DY, Růžičková, M: Controllability of linear discrete systems with constant coefficients and pure delay. SIAM J. Control Optim. 47, 1140-1149 (2008) 
11. Khusainov, DY, Diblík, J, Růžičková, M, Lukáčová, J: Representation of a solution of the Cauchy problem for an oscillating system with pure delay. Nonlinear Oscil. 11(2), 276-285 (2008)

12. Khusainov, DY, Shuklin, GV: Relative controllability in systems with pure delay. Int. Appl. Mech. 41, $210-221$ (2005)

13. Medved', M, Pospíšil, M: Sufficient conditions for the asymptotic stability of nonlinear multidelay differential equations with linear parts defined by pairwise permutable matrices. Nonlinear Anal., Theory Methods Appl. 75 , 3348-3363 (2012)

14. Medved', M, Pospíšil, M, Škripková, L: Stability and the nonexistence of blowing-up solutions of nonlinear delay systems with linear parts defined by permutable matrices. Nonlinear Anal., Theory Methods Appl. 74, 3903-3911 (2011)

15. Medved', M, Škripková, L: Sufficient conditions for the exponential stability of delay difference equations with linear parts defined by permutable matrices. Electron. J. Qual. Theory Differ. Equ. 2012, 22 (2012)

16. Pospísil, M: Representation and stability of solutions of systems of functional differential equations with multiple delays. Electron. J. Qual. Theory Differ. Equ. 2012, 4 (2012)

doi:10.1186/1687-1847-2013-139

Cite this article as: Diblík and Morávková: Discrete matrix delayed exponential for two delays and its property.

Advances in Difference Equations 2013 2013:139.

\section{Submit your manuscript to a SpringerOpen ${ }^{\circ}$ journal and benefit from:}

- Convenient online submission

- Rigorous peer review

- Immediate publication on acceptance

Open access: articles freely available online

- High visibility within the field

- Retaining the copyright to your article 